\title{
MUJERES RURALES COLOMBIANAS COMO GRUPO VULNERABILIZADO EN EL MARCO DE LA LEY DE VÍCTIMAS Y \\ RESTITUCIÓN DE TIERRAS \\ Ley 1448 de 2011
}

\author{
COLOMBIAN RURAL WOMEN AS A VULNERABLE GROUP WITHIN THE \\ FRAMEWORK OF THE LAW ON VICTIMS AND LAND RESTITUTION \\ Law 1448 of 2011
}

Angie Valentina Arango Delgado*

RESUMEN: Este trabajo propone explicar, a partir de la metodología propuesta por la Comisión Europea en el "Manual para la Perspectiva de género", por qué las mujeres rurales víctimas del conflicto armado en Colombia se constituyen como un grupo especialmente vulnerabilizado y cuáles son las principales barreras a las que se enfrentan al momento de reclamar su derecho a la restitución de tierras, específicamente en los programas que se vienen desarrollando en el marco de la Ley 1448 de 2011.

ABSTRACT: This paper proposes to explain, based on the methodology proposed by the European Commission in the "Gender Mainstreaming Manual", why rural women victims of the armed conflict in Colombia are a particularly vulnerable group and what are the main barriers they face when claiming their right to land restitution, specifically in the programmes that are being developed within the framework of Law 1448 of 2011.

PALABRAS CLAVE: mujeres rurales, desigualdad estructural, acceso a la tierra, perspectiva de género.

KEY WORDS: rural women, structural inequality, access to land, gender mainstreaming.

Fecha de recepción: 01/11/2021

Fecha de aceptación: 01/11/2021

doi: https://doi.org/10.20318/universitas.2022.6584

\footnotetext{
* Licenciada en Derecho por la Universidad Libre en Colombia. Magíster en Estudios Avanzados en Derechos Humanos de la Universidad Carlos III de Madrid. E-mail: avarangodel24@gmail.com
} 


\section{1.-INTRODUCCIÓN}

En Colombia a partir del reconocimiento de la existencia de un conflicto armado interno que tiene más de 60 años y persiste todavía, se han adelantado algunas medidas legislativas, judiciales y de políticas públicas con el fin de propender por la reivindicación de los derechos humanos de las víctimas y colectivos desfavorecidos. En este escenario surge la Ley 1448 de 2011, que se reconoce como un avance importante en el reconocimiento del enfoque diferencial de género de un sector excluido e invisibilizado como han sido las mujeres rurales, ya que son las que han padecido en carne propia todos los impactos de la guerra, que se manifiestan en violencia sobre sus cuerpos, su vida, su familia y su entorno.

En este sentido el presente escrito pretende explicar por qué las mujeres rurales víctimas del conflicto armado se constituyen como un grupo especialmente vulnerabilizado y cuáles son las principales barreras a las que se enfrentan al momento de reclamar su derecho a la restitución de tierras, específicamente en los programas que se vienen desarrollando en el marco de la Ley 1448 de 2011.

Este análisis resulta muy útil de cara a la implementación del Acuerdo Final Para La Terminación Del Conflicto y La Construcción De Una Paz Estable y Duradera, pues en el punto 1 se acordó la Reforma Rural Integral, la cual resalta el rol esencial de las mujeres en la economía rural e integra el enfoque de género como uno de los principios no sólo de la Reforma Rural Integral sino de todos los puntos del Acuerdo, en aras de dar solución a las necesidades estructurales de las mujeres rurales en Colombia.

Por esta razón la experiencia en el desarrollo normativo de la Ley de Restitución de Tierras y en su implementación puede evidenciar las problemáticas y los principales retos que se tienen en la restitución de tierras a las mujeres campesinas, y esto puede dar luces para que a través de las políticas que se establezcan derivadas del Acuerdo de Paz se mejoren todos estos aspectos que siguen siendo discriminatorios para las mujeres en el contexto rural.

La estructura del trabajo se construirá a partir de la metodología propuesta por la Comisión Europea en el "Manual para la Perspectiva de género" de la siguiente manera: i) presenta cuáles son las obligaciones internacionales del Estado colombiano en materia de la igualdad de las mujeres víctimas del conflicto en el acceso a la tierra; ii) muestra algunos datos y antecedentes que ayudan a reconocer las diferencias de género más relevantes que justifican las medidas de enfoque diferencial de la ley en cuestión; (iii) hace una breve descripción de las medidas diferenciales de género que contempla la ley con el objetivo de asegurar el derecho de las mujeres a la restitución de tierras; iv) evalúa el impacto de género de la ley en el grupo vulnerable específico; v) estudia formas para rediseñar la ley a 
través de la proposición de una serie de recomendaciones para que la igualdad de género y el enfoque diferencial se garanticen realmente.

\section{2.- MAINSTREAMING DE GÉNERO EN LA LEY 1448 DE 2011}

\section{1.- Marco jurídico nacional e internacional de protección de la igualdad de las mujeres rurales víctimas del conflicto armado}

Para desarrollar la estructura que se propone, resulta esencial en primer lugar determinar cuáles son las obligaciones que tiene el Estado colombiano en materia del derecho a la igualdad de las mujeres rurales víctimas del conflicto armado en lo relacionado con el acceso a la tierra, lo anterior a la luz del marco jurídico nacional e internacional.

De manera general, los derechos de las mujeres rurales, incluyendo la igualdad en el acceso a los recursos como la tierra, se encuentran concebidos en diversos instrumentos internacionales de carácter vinculante para el Estado Colombiano como se verá a continuación.

En el caso del "Pacto Internacional de Derechos Civiles y Políticos"1 y el "Pacto Internacional de Derechos Económicos, Sociales y Culturales"2, se incluye la cláusula general de no discriminación por medio de la cual "los Estados se comprometen a respetar y garantizar a todos los individuos que se encuentren en su territorio y estén sujetos a su jurisdicción los derechos reconocidos en dichos Pactos, sin distinción alguna de raza, color, sexo, idioma, religión, opinión política o de otra índole, origen nacional o social, posición económica, nacimiento o cualquier otra condición social" 3 .

Resaltando las categorías que son de mayor interés para el objeto de este trabajo, es decir, la categoría del sexo, el origen nacional o social y la posición económica, a través de estos Pactos también se establece la obligación de los Estados de adelantar medidas afirmativas para que la igualdad formal, bajo estos condicionamientos sociales especiales, se traduzca en igualdad material.

Más adelante, en la "Convención contra Todas las Formas de Discriminación contra la Mujer" 4 , ratificando la cláusula de no

\footnotetext{
${ }^{1}$ Asamblea General de las Naciones Unidas, Pacto Internacional de Derechos Civiles $y \quad$ Políticos (1976) art 2 $<$ www.ohchr.org/Documents/ProfessionalInterest/ccpr SP.pdf> accedido el 8 de septiembre de 2021.

2 Asamblea General de las Naciones Unidas, Pacto Internacional de Derechos Económicos, Sociales $y \quad$ Culturales (1966) art $2<$ www.ohchr.org/sp/professionalinterest/pages/cescr.aspx $>$ accedido el 8 de septiembre de 2021.

3 Ibid., art 2.

${ }^{4}$ Asamblea General de las Naciones Unidas, Convención sobre la eliminación de todas las formas de discriminación contra la mujer(1979) art 1 $<$ www.ohchr.org/sp/professionalinterest/pages/cedaw.aspx $>$ accedido el 8 de septiembre de 2021.
} 
discriminación en razón del sexo contemplada en los Pactos mencionados y exigiendo la adecuación de las normatividades nacionales a dicha cláusula ${ }^{5}$, se expone lo siguiente:

"La CEDAW hace un reconocimiento específico a los derechos de las mujeres rurales, tales como la participación en la formulación e implementación de los planes de desarrollo y en las actividades comunitarias, y el accesoa la seguridad social, los servicios de salud, la educación y capacitación técnica. Igualmente, la CEDAW establece que las mujeres rurales deben contar con garantías para el acceso a créditos, comercializacióny tecnologías apropiadas y al factor más importante en el campo, a recibir trato igual en la asignación y distribución de tierras (Artículo 14). A propósito del derecho a la propiedad, la CEDAW establece la igualdad de mujeres y hombres, incluyendo lo relacionado con la firma de contratos y la administración de bienes (Artículo 15). Además, reconoce la igualdad de hombres y mujeres en la familia y el matrimonio, estableciendo que los Estados deben tomar medidas para eliminar la discriminación en el matrimonio y las relaciones familiares, asegurando el derecho de las mujeres a gozar de los mismos derechos y responsabilidades que los hombres, tanto durante el matrimonio, como cuando éste se acabe (Artículo 16). Este derecho cobra importancia para que los derechos de propiedad de las mujeres se garanticen cuando el matrimonio se disuelve y en las situaciones de desplazamiento forzado" 6 .

Por su parte, la Comisión de Derechos Humanos de Naciones Unidas recomienda a los Estados afianzarla igualdad de las mujeres en el acceso a los programas de reforma agraria ${ }^{7}$. La Comisión de Derechos Humanos reconoció que "las leyes, políticas, costumbres y tradiciones que restringen a las mujeres el acceso igualitario al crédito y los préstamos, les impiden además adquirir y heredar tierras, propiedadesy vivienda, y las excluyen de una plena participación en el proceso de desarrollo son discriminatorias y pueden contribuir a la

\footnotetext{
${ }^{5}$ Ibid., art 2 (a) "a) Consagrar, si aún no lo han hecho, en sus constituciones nacionales y en cualquier otra legislación apropiada el principio de la igualdad del hombre y de la mujer y asegurar por ley u otros medios apropiados la realización práctica de ese principio"

${ }^{6}$ Comisión Colombiana de Juristas, La política agraria y los derechos de las mujeres en Colombia (2011) p.

$<$ www.coljuristas.org/documentos/libros e informes/la politica agraria y los der echos de las mujeres.pdf> accedido el 8 de septiembre de 2021.

7 Consejo Económico y Social, Resolución de la Comisión de Derechos Humanos 2000/13. La igualdad de las mujeres en materia de propiedad, acceso y control de la tierra y la igualdad de derechos a la propiedad y a una vivienda adecuada (2000) núm. $5<$ www.acnur.org/fileadmin/Documentos/BDL/2002/0653.pdf?file=fileadmin/Docu mentos/BDL/2002/0653. Numeral\%205.> accedido el 8 de septiembre de 2021.
} 
feminización de la pobreza"8. En razón a lo anterior, los Estados deben promover procesos culturales de modificación de tradiciones y costumbres que implican una discriminación hacia la mujer y les deniega un tratamiento igualitario en todo lo que tiene que ver con el acceso a la tierra, la disponibilidad, la tenencia y control sobre la misma, en especial en este contexto de las mujeres rurales en el cual la mayoría están en situación de extrema pobreza y son madres cabeza de familia.

Se identifican paralelamente unos instrumentos internacionales que hacen mención específicamente al grupo de las mujeres rurales víctimas del conflicto armado y su derecho a la restitución de tierras, estos son los "Principios sobre la restitución de las viviendas y el patrimonio de los refugiados y las personas desplazadas" ${ }^{9}$. En particular:

"estos Principios reconocen el derecho a la igualdad entre hombres y mujeres en el goce del derecho a la restitución y que los Estados tiene la obligación de garantizar la igualdad en el goce de los derechos al retorno voluntario en condiciones de seguridad y dignidad; a la seguridad jurídica de la tenencia; a la propiedad del patrimonio; a la sucesión; y al acceso, uso y control de las tierras, las viviendas y el patrimonio. Esta igualdad debe ser garantizada en las políticas y programas de restitución de la incorporación de un enfoque de género y del reconocimiento explícito de la titularidad conjunta de ambas cabezas de familia, (Principio n.$^{\circ} 4$ ). En materia de restitución de las viviendas y el patrimonio, los Estados están obligados a revocar las leyes que generen efectos discriminatorios y a garantizar que sus políticas de restitución garanticen plenamente a las mujeres la protección contra la discriminación y a la igualdad de hecho y de derecho (Principio $n .^{\circ}$ 9)"10.

Asimismo, se resalta la "Declaración de las Naciones Unidas sobre los Derechos de los Campesinos y de Otras Personas que Trabajan en las Zonas Rurales"11 en la cual se reconoce que "las campesinas a otras mujeres de las zonas rurales desempeñan un papel importante en la supervivencia económica de su familia a mediante su

\footnotetext{
8 Ibid., párr. 5

9 Organización de las Naciones Unidas, Principios sobre la restitución de las viviendas $y$ el patrimonio de los refugiados $y$ las personas desplazadas (2007) < www.acnur.org/fileadmin/Documentos/Publicaciones/2008/63 25.pdf> accedido el 8 de septiembre de 2021.

${ }^{10}$ Comisión Colombiana de Juristas, Óp. Cit., p. 50.

${ }^{11}$ Asamblea General de las Naciones Unidas, Declaración de las Naciones Unidas sobre los Derechos de los Campesinos y de Otras Personas que Trabajan en las Zonas Rurales (2018) < www.ohchr.org/Documents/HRBodies/HRCouncil/WGPleasants/AHRC-WG-15-1-2 sp.pdf> accedido el 8 de septiembre de 2021.
} 
contribución a la economía rural a nacional, en particular por su trabajo en los sectores no monetarios de la economía, pero que a menudo se les niega la tenencia a propiedad de la tierra a el acceso en condiciones de igualdad a la tierra, los recursos productivos, los servicios financieros, la información, el empleo o la protección social, a con frecuencia son víctimas de la violencia a la discriminación en diversas formas y manifestaciones"12. En este sentido, se deriva de la aplicación de la mencionada Declaración, la obligación para los Estados de prestar especial atención a las necesidades y derechos de las mujeres rurales, tanto así que en su artículo 4 contempla de manera expresa diez derechos que se consideran como los más importantes para estas mujeres y que a su vez los más transgredidos. Entre estos, se incluye el derecho de acceso a la tierra y a los recursos naturales en igualdad de condiciones, el derecho a la participación en los planes de desarrollo, el derecho a tener un empleo decente y acceso a la seguridad social, y por último a tener una vida libre de todas las formas de violencia ${ }^{13}$. Es necesario resaltar que, en el año 2018, el Estado colombiano se abstuvo de firmar esta Declaración, a pesar de que era completamente compatible con la Constitución Nacional y el desarrollo jurisprudencial de la Corte Constitucional ${ }^{14}$.

Así las cosas, las disposiciones mencionadas, conforme al bloque de constitucionalidad, se encuentran traducidas en la legislación nacional colombiana de la siguiente manera:

La Carta Política del 91 indica de manera general las responsabilidades del Estado Colombiano respecto de los derechos económicos, sociales y culturales de la población rural y también concibe obligaciones específicas en favor de las mujeres rurales, en especial las madres cabeza de familia.

Según la Constitución Política "es deber del Estado promover el acceso progresivo a la propiedad de la tierra de los trabajadores agrarios, en forma individual o asociativa, y a los servicios de educación, salud, vivienda, seguridad social, recreación, crédito, comunicaciones, comercialización de los productos, asistencia técnica y empresarial, con el fin de mejorar el ingreso y calidad de vida de la población

\footnotetext{
12 Ibid., p. 3.

13 Ibid., art. 4.

${ }^{14} \mathrm{Si}$ bien el documento no es de obligatorio cumplimiento, puede jugar un papel fundamental como herramienta de principios y parámetros de interpretación a la hora de tomar decisiones judiciales, legislativas y/o comunitarias que afecten a la población campesina en Colombia. Así lo sugirió el Comité para la Eliminación de la Discriminación contra la Mujer, en el capítulo de las recomendaciones sobre mujeres rurales para el Estado Colombiano CEDAW/C/COL/CO/9: "Considere la posibilidad de incorporar los principios contenidos en la Declaración de las Naciones Unidas sobre los Derechos de los Campesinos y de Otras Personas que Trabajan en las Zonas Rurales en los instrumentos jurídicos y de políticas relativos a las mujeres rurales".
} 
campesina"15. Contempla la igualdad como valor, principio y derecho fundamental conforme a la cláusula general de no discriminación y de igualdad formal y material entre hombres y mujeres en materia de derechos y oportunidades. En el mismo sentido reconoce a las mujeres cabeza de familia como sujetos de especial protección constitucional.

Asimismo, de acuerdo con el mandato de la Corte Constitucional de interpretar la Carta Fundamental, se ha desarrollado ampliamente el tema del enfoque diferencial de género necesario para analizar la situación de vulnerabilidad del grupo de las mujeres rurales víctimas del conflicto armado y su derecho a la restitución de tierras ${ }^{16}$. Así por ejemplo, a través del Auto 218 de $2006^{17}$, que es un auto de seguimiento a la Sentencia T-025 de $2004^{18}$, se llama la atención sobre la ausencia del enfoque diferencial de cara a la política pública de víctimas de desplazamiento forzado, señalando tres niveles: "(a) en relación con los sujetos de especial protección constitucional que forman parte de la población desplazada -ancianos, niños, mujeres cabeza de familia-, (b) en relación con las diferenciales regionales que presenta el fenómeno del desplazamiento, y (c) en relación con la condición de víctimas del conflicto armado que tienen las personas desplazadas"19.

Frente al primer criterio de especificidad, referente a los distintos grupos de población vulnerable, se destaca que "los sujetos de especial protección constitucional se diferencian del resto (de la población desplazada) en cuanto a la especificidad de sus vulnerabilidades, sus necesidades de protección y de atención, y las posibilidades que tienen de reconstruir sus proyectos de vida digna"20.

15 Constitución Política de Colombia, 13 de junio de 1991, art. 13-43$64<$ www.secretariasenado.gov.co/senado/basedoc/constitucion politica 1991.html> accedido el 8 de septiembre de 2021.

${ }^{16}$ La Corte Constitucional se ha referido sobre la importancia de la incorporación del enfoque diferencial y de género en Autos como el 092 de 2008, 237 de 2008, 009 de 2015, 373 de 2016, y 737 de 2017.

17 Auto 218/2006, Corte Constitucional, 11 de agosto de 2006 (Colombia) XXXX <www.corteconstitucional.gov.co/T-025-

04/AUTOS\%202006/26.\%20Auto\%20del\%2011-08-

2009.\%20Auto\%20218.\%20Verificacion\%20medidas.PDF> accedido el 8 de septiembre de 2021.

18 Sentencia T024-2004, Corte Constitucional, 21 de enero de 2004 (Colombia) <www.corteconstitucional.gov.co/relatoria/2004/T-024-04.htm>

accedido el 8 de septiembre de 2021: por medio de la cual "se declara la existencia de un estado de cosas inconstitucional en la situación de la población desplazada debido a la falta de concordancia entre la gravedad de la afectación de los derechos reconocidos constitucionalmente y desarrollados por la ley, de un lado, y el volumen de recursos efectivamente destinado a asegurar el goce efectivo de tales derechos y la capacidad institucional para implementar los correspondientes mandatos constitucionales y legales, de otro lado".

19 Auto 218/2006, Óp. Cit., núm. 5.2.

20 Ibid., núm. 5.3.1. 
En efecto, atendiendo a este criterio del impacto diferenciado del conflicto armado sobre algunos sujetos de especial protección constitucional, la misma Corte, a través del Auto 092 de 2008, "ordenó a todas las autoridades considerar la afectación diferencial y desproporcionada del conflicto armado en la vida de las mujeres para el diseño e implementación de políticas públicas para su atención, protección y reparación, así como para la prevención de la continuidad de violencias, con un reconocimiento especial a la situación de mujeres indígenas y afro descendientes, por su situación de mayor vulnerabilidad"21.

En dicho auto también se exponen diez factores específicos de vulnerabilidad que hacen que las mujeres sean impactadas de manera desproporcionada en comparación con los hombres, por causa del fenómeno del desplazamiento forzado. Entre ellos se destaca, "el riesgo de ser despojadas de sus tierras y su patrimonio con mayor facilidad por los actores armados ilegales dada su posición histórica ante la propiedad, especialmente la propiedad de inmuebles rurales"22. También se identifican "dieciocho (18) facetas de género del desplazamiento forzado" 23 , de las que se resaltan: "obstáculos agravados en la inserción al sistema económico y en el acceso a oportunidades laborales y productivas"; "la explotación doméstica y laboral, incluida la trata de personas con fines de explotación económica"; "obstáculos agravados en el acceso a la propiedad de la tierra y en la protección de su patrimonio hacia el futuro, especialmente en los planes de retorno y reubicación" 24 .

Pues bien, a raíz de todo este desarrollo normativo expuesto, y con el fin de adecuar los estándares internacionales y constitucionales en materia de protección específica a las mujeres rurales víctimas del conflicto armado y su acceso a los diferentes derechos, incluido el acceso a la tierra, se han promulgado una larga lista de Leyes, entre las que se distinguen las siguientes: la Ley 731 de 2002 que está enfocada en mejorar la calidad de vida de las mujeres rurales en situación de pobreza, y la cual no ha sido todavía reglamentada; la Ley 1257 de 2008 trata sobrela prevención y sanción de la violencia y

21 Auto 092/2008, Corte Constitucional, 14 de abril de 2008 (Colombia) II. 2 <www.corteconstitucional.gov.co/relatoria/autos/2008/a092-

08.htm > accedido el 8 de septiembre de 2021.

22 Ibid., literal c.

23 "Aspectos del desplazamiento que impactan de manera diferencial, específica y agudizada a las mujeres, por causa de su condición femenina en el marco del conflicto armado colombiano. Entre estas facetas se incluyen: (A) patrones de violencia y discriminación de género de índole estructural en la sociedad colombiana, preexistentes al desplazamiento pero que se ven potenciados y degenerados por el mismo, impactando en forma más aguda a las mujeres desplazadas, y (B) problemas específicos de las mujeres desplazadas, producto de la conjunción de los factores de vulnerabilidad que soportan, y que no afectan ni a las mujeres no desplazadas, ni a los hombres desplazados".

${ }^{24}$ Auto 092/2008, Óp. Cit., literal d. 
discriminación contra las mujeres que comprende el daño patrimonial de las mujeres campesinas; y la Ley de Víctimas y Restitución de Tierras $^{25}$ que incluye en sus principios el enfoque diferencial y que se constituye como el objeto de estudio del presente escrito. Aclarando al respecto que la última Ley mencionada, esta es, la Ley 1448 de 2011, tenía una vigencia de 10 años que iba hasta el día 10 de junio de 2021. Sin embargo, fue prorrogada hasta el 10 de junio de 2031 por medio de la Ley 2078 de $2021^{26}$, por tanto, es la legislación que se sigue aplicando en materia de reparación de víctimas y restitución de tierras en el marco del conflicto armado en Colombia, y su análisis sigue siendo necesario y actual.

\section{2.- Conociendo las diferencias de género}

En este apartado se explicará por qué se considera que las mujeres rurales víctimas del conflicto armado en el contexto colombiano se constituyen como un grupo especialmente vulnerabilizado. Lo anterior atendiendo a los datos y estadísticas que exponen las características sociodemográficas de la población rural, también resaltando las diferencias que se presentan entre hombre y mujeres rurales al momento de acceder a recursos como la tierra y el uso del tiempo; y por último evidenciando como la existencia de patrones culturales de discriminación y estereotipos de género que permanecen en la cultura campesina, refuerzan el acceso desigual a los recursos mencionados y además generan situaciones de mayor exposición a la violencia para las mujeres.

Según proyecciones realizadas por el último censo poblacional realizado en el año 2018 por el DANE ${ }^{27}$, Colombia tenía 49.395.678 de habitantes, de los cuales el $51,16 \%$ son mujeres. De igual manera, a través de la Encuesta de Cultura Política ${ }^{28}$ realizada por la misma entidad, la cual se constituye como la primera radiografía general que se realiza de la población campesina, se encontró que el total de esta población asciende a la cifra de 11.969 .822 personas, que se constituye como el $24,2 \%$ del total de la población del país.

Las mujeres campesinas ascienden a 5.760 .524 , son el $48.13 \%$ de la población rural, mientras que los hombres son el $51.87 \%$, es decir

25 Ley 1448 de 2011, 10 de junio de 2011 (Colombia) <http://wp.presidencia.gov.co/sitios/normativa/leyes/Documents/Juridi ca/LEY\%201448\%20DE\%202011.pdf> accedido el 8 de septiembre de 2021.

26 Ley 2078 de 2021, 8 de enero de 2021 (Colombia) <https://dapre.presidencia.gov.co/normativa/normativa/LEY\%202078\%20DEL\%20 8\%20DE\%20ENERO\%20DE\%202021.pdf> accedido el 8 de septiembre de 2021

27 "Censo Nacional de Población y Vivienda" (DANE, 10 de octubre de 2018) $<$ www.dane.gov.co/> accedido el 8 de septiembre de 2021.

28 "Encuesta de cultura política (ECP)" (DANE, 8 de noviembre de 2019) $<$ www.dane.gov.co/index.php/comunicados-y-boletines/estadisticas-

sociales/cultura-politica > accedido el 8 de septiembre de 2021. 
no son mayoría como sucede en el escenario urbano ${ }^{29}$. De este total de mujeres rurales, 1.232.417 mujeres se autoreconocen como pertenecientes a algún grupo étnico. Así las cosas, del total de las mujeres rurales, el $10 \%$ son negras, mulatas, afrodescendiente 0 afrocolombianas, $15,5 \%$ son mujeres indígenas, el $0,13 \%$ son mujeres raizales del Archipiélago de San Andrés y Providencia, el 0,002\% son gitanas rom, el 0,031\% son palenqueras san basilio o descendiente ${ }^{30}$.

Las mujeres en Colombia han sido además inmensamente afectadas por el conflicto armado. Según el Registro Único de Víctimas, al año 2021 se reportan un total de 9.165.126 víctimas, de las cuales 4.476.658 son mujeres que corresponden al 48,84\% de la población afectada $^{31}$. Concretamente, se identifican como mujeres víctimas de abandono y despojo forzado de tierras un total de 14.926, mientras que los hombres son 15.180. Asimismo, para los delitos contra la libertad y la integridad sexual en desarrollo del conflicto armado el número de mujeres víctimas asciende a 30.283, y los hombres víctimas son $2.793^{32}$.

Con este panorama general, se puede observar que, de acuerdo con las estadísticas, las mujeres y en especial las mujeres rurales, no son un grupo significativamente minoritario en ninguno de los aspectos que se resaltan para efectos de este trabajo. Sin embargo, su situación de vulnerabilidad y su definición como "grupo minoritario", están determinadas por razón de su posición de subordinación social, política y económica, subordinación causada por la discriminación estructural que se agrava en el escenario del conflicto armado.

A nivel general, en relación con todos los indicadores de género y desarrollo para los que existen datos, las mujeres rurales colombianas se encuentran en peor situación que los hombres rurales y las mujeres y los hombres urbanos, y la pobreza y exclusión les afectan de manera desproporcionada.

\footnotetext{
29 "Nota estadística: Mujeres Rurales en Colombia" (DANE, 27 de noviembre de 2020) $<$ www.dane.gov.co/index.php/estadisticas-por-tema/enfoque-diferencial-einterseccional/enfoque-de-genero> accedido el 8 de septiembre de 2021.

${ }^{30}$ Esta última caracterización por etnias es relevante pues en el marco de la Ley de Restitución de Tierras se diferencia a la población campesina de los pueblos indígenas y afrodescendientes, con los cuales se realizan restituciones colectivas y por tanto allí las estadísticas con perspectiva de género en materia de restitución de tierras son inexistentes.

31 "Registro Único de Víctimas" (Unidad para la Reparación y Atención Integral a las Víctimas, 31 de julio de 2021) <www.unidadvictimas.gov.co/es/registro-unico-devictimas-ruv/37394> accedido el 8 de septiembre de 2021.

32 Poner en relación el número de víctimas con respecto de los delitos contra la libertad e integridad sexual es relevante aquí pues en materia de restitución de tierras, en muchas ocasiones, no se toman en cuenta las afectaciones particulares de las mujeres cuando son víctimas de violencia sexual y cuando ésta es la causa principal del despojo y el desplazamiento.
} 
De acuerdo con la Recomendación general número 34 sobre los derechos de las mujeres rurales, emitida por el Comité para la Eliminación de la Discriminación contra la Mujer, las mujeres rurales:

"se enfrentan a una discriminación sistemática en el acceso a la tierra y los recursos naturales. Soportan la mayor parte de la carga de trabajo no remunerado debido a los papeles estereotipados asignados a cada género, la desigualdad dentro del hogar y la falta de infraestructura y servicios, también con respecto a la producción alimentaria y el trabajo asistencial. Aun cuando tienen un empleo formal, desempeñan con mayor frecuencia trabajos inseguros, peligrosos, mal remunerados y no cubiertos por la protección social. Es menos probable que hayan recibido educación y corren mayor riesgo de ser víctimas de la trata y el trabajo forzoso, así como el matrimonio infantil $\mathrm{y} / \mathrm{o}$ forzado y otras prácticas nocivas (véase CEDAW/C/GC/31-CRC/C/GC/18). Tienen más probabilidades de caer enfermas, sufrir malnutrición o morir por causas prevenibles, y sufren especial desventaja con respecto al acceso a la atención sanitaria. Las mujeres rurales también tienen más probabilidades de verse excluidas de los puestos de liderazgo y toma de decisiones a todos los niveles. Se ven afectadas de manera desproporcionada por la violencia por razón de género y la falta de acceso a la justicia y a recursos jurídicos eficaces."33

Esta Recomendación a pesar de dar cuenta de la situación general de las mujeres rurales en todo el mundo, muestra muy bien la realidad de las mujeres rurales en Colombia. Conforme con todos los factores de discriminación sistemática enunciados, se resaltarán dos recursos que se consideran como los más importantes y definitorios en la perpetuación de la desigualdad estructural de las mujeres, estos son, el acceso desigual de la tierra y la división sexual del trabajo.

\subsection{1. - Mujeres y acceso desigual a la tierra}

En Colombia, la alta concentración de la tierra en manos de unos pocos ha sido uno de los más fuertes obstáculos para el desarrollo humano. Así lo ha reconocido el PNUD, que para el 2011 midió el índice Gini de concentración de propiedad rural en 0,86, ubicando a Colombia en la lista de los países con mayor desigualdad en el acceso a la tierra en el mundo ${ }^{34}$. El $1 \%$ de las fincas más grandes ocupan el $81 \%$ de ${ }^{33}$ Recomendación general núm. 34 (2016) sobre los derechos de las mujeres
rurales (CEDAW/C/GC/34,
2016 ) p.4 <www.acnur.org/fileadmin/Documentos/BDL/2016/10709.pdf?file=filead
min/Documentos/BDL/2016/10709> accedido el 8 de septiembre de 2021.
34 Programa de las Naciones Unidas para el Desarrollo, Informe Nacional de
Desarrollo 
Colombia. El 80 \% de la tierra con uso agropecuario está dedicado a la ganadería y solo el $20 \%$ a la agricultura ${ }^{35}$. Pues bien, desde las organizaciones de mujeres campesinas, se denuncia que en Colombia "tiene más derechos una vaca que una mujer, porque nosotras no tenemos tierra ni en las uñas" ${ }^{36}$, afirmación que está muy bien sustentada en los datos referidos anteriormente, y además teniendo en cuenta que según la Organización International Land Coalition, las mujeres en Colombia sólo tienen titularidad sobre el $26 \%$ del total de las tierras ${ }^{37}$.

La desigualdad en la distribución de las tierras en Colombia es un problema tan grave y extendido en el tiempo que ha sido reconocida como una de las causas estructurales del origen del conflicto armado. Este escenario impacta de manera diferencial a las mujeres rurales, pues son ellas las que como consecuencia de la desigualdad social histórica y la discriminación de género que prevalece en la cultura agraria patriarcal que "tradicionalmente ha privilegiado valores y estereotipos masculinos reforzados pordécadas de conflicto armado"38, sufren de manera excesiva los efectos del acceso desigual a la tierra y a los recursos relacionados con esta, lo que afecta evidentemente su productividad en este sector, generando de ese modo una brecha de productividad con respecto a los hombres.

Así se ve reflejada en la misma radiografía realizada por Oxfam, al afirmar que existen grandes brechas de género en el sector agropecuario, pues mientras que el " $26 \%$ de las explotaciones de la tierra manejadas por personas naturales está a cargo de mujeres, el $61 \%$ está a cargo de hombres y el restante $12,6 \%$ es manejada de forma mixta"39.

2011 (2011) < www1.undp.org/content/undp/es/home/librarypage/hdr/human dev elopmentreport2011.html> accedido el 8 de septiembre de 2021.

35 OXFAM, Radiografía de la desigualdad. Lo que nos dice el último censo agropecuario sobre la distribución de la tierra en Colombia (2017)

$<$ https://oi-files-d8-prod.s3.eu-west-2.amazonaws.com/s3fs-

public/file attachments/radiografia de la desigualdad.pdf $>$ accedido el 8 de septiembre de 2021.

36 "En Caquetá Tiene Más Derechos Una Vaca Que Una Mujer": La Lucha Por La Tierra De Las Lideresas Campesinas En Colombia' (2021) Mongabay- Periodismo Ambiental Independiente en América Latina <https://es.mongabay.com/2021/03/en-caquetatiene-mas-derechos-una-vaca-que-una-mujer-la-lucha-por-la-tierra-de-las-

lideresas-campesinas-en-colombia/> accedido el 8 de septiembre de 2021.

37 "Infografía Las Mujeres Rurales en América Latina" (International Land Coalition, 12 de diciembre de 2018) $<$ https://lac.landcoalition.org/es/recursos/infograf\%C3\%ADa-las-mujeres-ruralesen-am\%C3\%A9rica-latina/> accedido el 8 de septiembre de 2021.

${ }^{38} \mathrm{Al}$ respecto revisar la jurisprudencia de la Corte Constitucional que ha reconocido en varias sentencias y autos, particularmente el 092 de 2008 y el 004 de 2009 que describen los impactos diferenciales del conflicto armado en las mujeres rurales por su condición de género y etnia, no compartidos por los hombres.

39 OXFAM, Óp. Cit., p. 20. 
Estas diferencias que se dan en el sector productivo responden en buena medida a las desventajas que tienen las mujeres en el acceso a la propiedad de la tierra, a los créditos, el uso de fertilizantes y semillasy la disponibilidad de mano de obra. Además, se suman como desventajas los estereotipos de género que se traducen en menores oportunidades para las mujeres de acceder a la tierra. Según el informe Voces del Cocal realizado por la organización De Justicia se pueden identificar los siguientes estereotipos de género: "i) a la hora de heredar hay preferencias por los hombres, al considerar que ellos formarán familia y necesitan tierra para producir; en cambio, las mujeres migrarán a la tierra en donde estén sus parejas. ii) Los privilegios de los hombres en el matrimonio y la cultura hacen que los mejores predios sean entregados a los varones iii) Los sesgos de género en el uso de la tierra comunal y iv) los sesgos masculinos en los programas de distribución y titulación de la tierra"40. Como consecuencia de las diferencias materiales y culturales, se ve limitada "la capacidad de actuación de las mujeres, su poder de toma de decisiones y su participación en el mercado de trabajo"41 y esto adicionalmente dificulta la transformación de los roles asignados tradicionalmente al hombre y la mujer por el modelo patriarcal predominante en el campo.

Para confirmar lo dicho se presenta el siguiente cuadro ilustrativo, para contextualizar la discriminación de las mujeres rurales colombianas en el acceso a los programas de adjudicación de tierras, con corte al 31 de marzo de 2021:

\begin{tabular}{|l|c|c|c|c|c|c|}
\hline \multirow{2}{*}{ BIENES } & \multicolumn{3}{|c|}{ ADJUDICACIONES \% } & \multicolumn{3}{c|}{ EXTENSIÓN DE TIERRA (HÉCTÁREAS) } \\
\cline { 2 - 7 } & MUJERES & HOMBRES & PAREJAS & MUJERES & HOMBRES & PAREJAS \\
\hline BALDÍOS & $30,4 \%$ & $69,3 \%$ & $0,3 \%$ & $340.579,712$ & $776.387,304$ & $3.003,7$ \\
\hline FISCALES & $43 \%$ & $57 \%$ & $36 \%$ & 878,11 & 1.164 & 735,12 \\
\hline PRIVADOS & $36 \%$ & $64 \%$ & $2,95 \%$ & $6.353,64$ & $11.295,36$ & 520,64 \\
\hline
\end{tabular}

Fuente: Cuadro de elaboración propia con cifras obtenidas de Procuraduría General de la Nación $2021^{42}$

\subsection{2.- División sexual del trabajo}

\footnotetext{
40 Ana Jimena Bautista Revelo y otras, Voces Desde El Cocal. Mujeres Que Construyen Territorio (De Justicia, 2018) p. 29.

41 Programa de las Naciones Unidas para el Desarrollo, Informe de Desarrollo $\begin{array}{llll}\text { Humano } & 2019 & \text { (2019) p. } & 184\end{array}$ <http://hdr.undp.org/sites/default/files/hdr_2019_overview_-_spanish.pdf> accedido el 8 de septiembre de 2021.

42 Procuraduría General de la Nación, Tercer Informe al Congreso sobre el estado de Avance de la Implementación del Acuerdo de Paz. Abril 2020-marzo 2021. $\begin{array}{lllll}\text { Conclusiones } y & \text { recomendaciones. } & \text { (2021) } & \text { p. }\end{array}$ $<$ https://www.procuraduria.gov.co/portal/media/file/Procuraduri\%CC $\% 81$ a $\% 20$ Con clusiones\%20y\%20recomendaciones\%20Tercer\%20Informe\%20Paz\%202021.pdf> accedido el 8 de septiembre de 2021.
} 
En las sociedades occidentales-patriarcales ha persistido la división sexual del trabajo; de acuerdo con ella, las mujeres han sido asignadas como responsables del trabajo reproductivo ${ }^{43}$, y los hombres del productivo ${ }^{44}$. Desde la economía feminista se critica esta asignación, pues la naturalización del trabajo reproductivo y su exclusión de la economía convencional han permitido que a las mujeres se les imponga toda la carga de las tareas de cuidado y sostenimiento de la vida sin que estas sean valoradas dentro del modelo capitalista, -pues no se considera otro trabajo diferente al mercantil, el trabajo que se compra y se vende por un salario- lo que contribuye a perpetuar las condiciones de opresión y desigualdad.

En Colombia, la distribución inequitativa entre hombres y mujeres del trabajo de cuidados en el hogar está muy documentada por cifras presentadas por el DANE, por ejemplo, a través de la ENUT que se muestra a continuación ${ }^{45}$. La misma encuesta indicó que el 12,7\% de las mujeres sintieron que el tiempo no les alcanzó para realizar todas sus tareas, frente al $8,1 \%$ de los varones 46.

43 Se refiere a todas las actividades y prácticas necesarias para la supervivencia cotidiana de las personas [...] incluye el autocuidado, el cuidado directo de otras personas, la provisión de las precondiciones en que se realiza el cuidado (la limpieza de la casa, la compra y preparación de alimentos) y la gestión del cuidado (coordinación de horarios, traslados a centros educativos y a otras instituciones, supervisión del trabajo de cuidadoras remuneradas, entre otros.

${ }^{44}$ Se remite a aquellas "actividades de producción de bienes y servicios para el mercado".

45 "Encuesta nacional del Uso del Tiempo" (DANE, 21 de junio de 2021) $<$ https://www.dane.gov.co/index.php/estadisticas-por-tema-2/pobreza-y-

condiciones-de-vida/encuesta-nacional-del-uso-del-tiempo-enut> accedido el 8 de septiembre de 2021.

46 Estas cifras se evidencian en múltiples testimonios de mujeres. Una lideresa de la comunidad de Subachoque, Cundinamarca señala que "uno no sabe cuánto es el tiempo de uno, entonces iaprovéchelo! Antes, cuando quería salir a caminar, lo hacía, pero ahora ya no puedo". Una de las razones de este fenómeno es que pese a que las mujeres están cada vez más presentes en el mercado laboral no dejan de realizar las tareas del hogar tradicionalmente asociadas a ellas. 


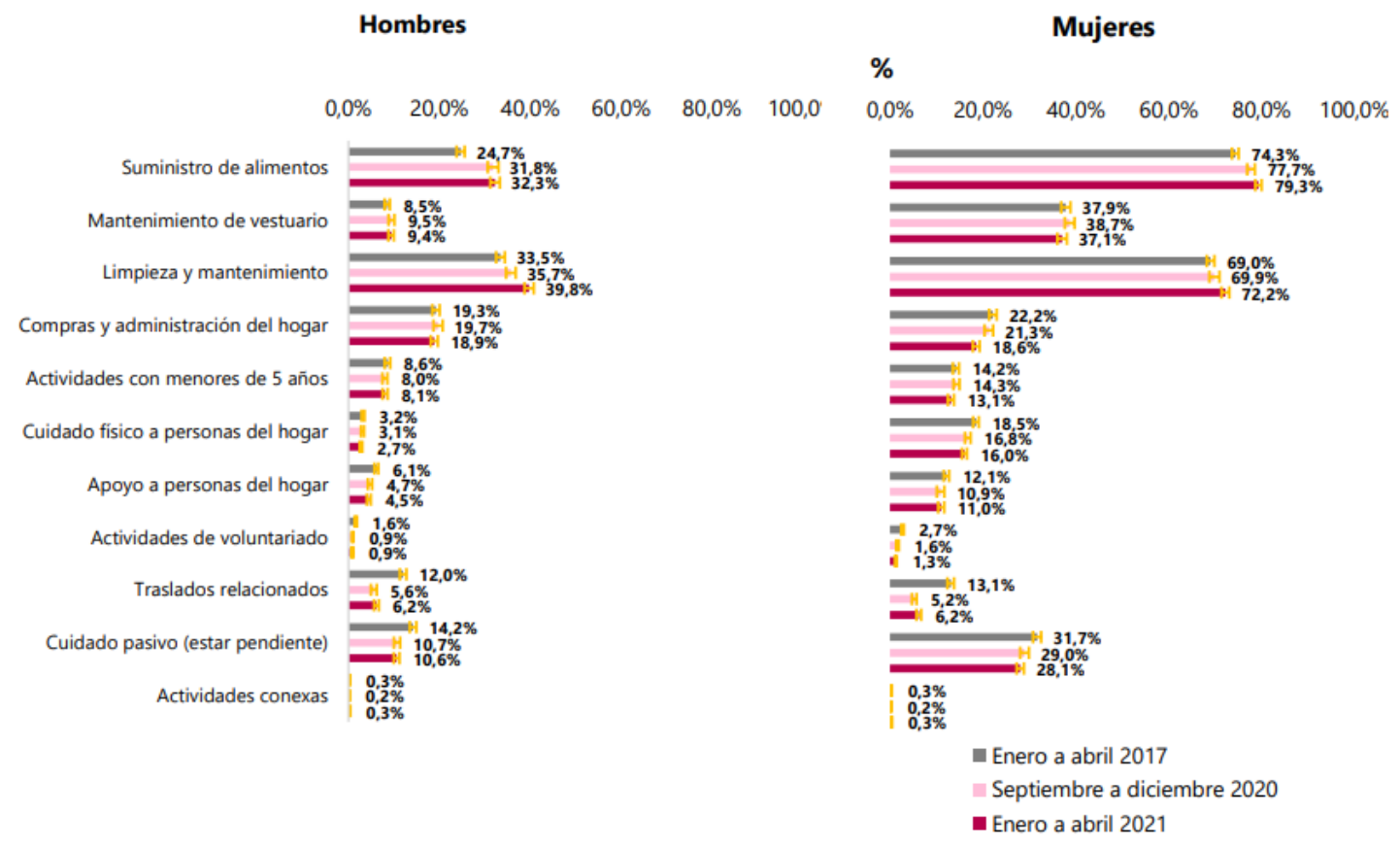

Fuente: DANE, Encuesta Nacional sobre el Uso del Tiempo 2021

En el sector específico de las mujeres rurales, según datos de la Encuesta de Cultura Política ${ }^{47}$ publicada en el año 2020, se observó la enorme brecha que existe entre mujeres y hombres rurales en materia del trabajo de cuidados u oficios del hogar. El DANE la denominó "población inactiva", señalando que en las "zonas rurales la mayoría de las mujeres inactivas $(65,8 \%)$ se dedican a oficios del hogar como actividad principal, mientras que este porcentaje es de $7,1 \%$ para los hombres. Contrariamente, la mayoría de los hombres inactivos $(65,7 \%)$ se encuentran estudiando como actividad principal, mientras que este porcentaje es de $26,9 \%$ para las mujeres" ${ }^{\prime 48}$.

Gráfica 10. Tipo de actividad de la población inactiva, según sexo (porcentaje)

Total centros poblados y rural disperso 2019

- Estudiando

- Oficios del hogar

- Otra actividad
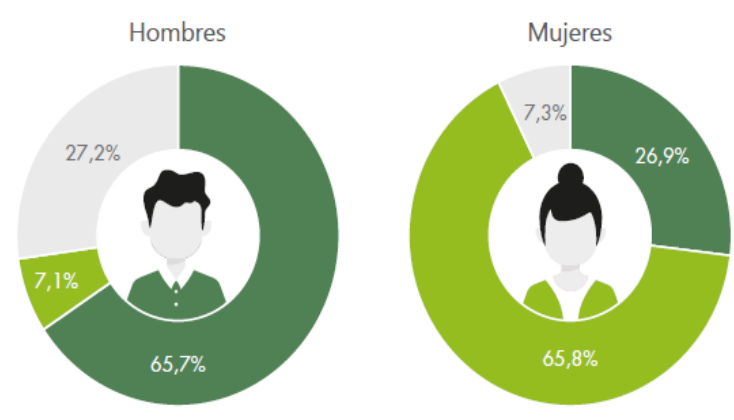

Fuente: DANE - GEIH, 2019.

47"Encuesta de cultura política (ECP)", Óp. Cit., apartado Identificación Subjetiva. Cuadro No. 13.

48 "Nota estadística: Mujeres Rurales en Colombia", Óp. Cit., p. 20. 
Así las cosas, si las cuentas nacionales de Colombia integraran en su medición el trabajo doméstico y de cuidados no remunerados, se encontraría que este es el sector que más participación tiene dentro de la economía, es decir, la rama de actividad que más aporta al PIB. Así quedó determinado en último informe del DANE en el que se realizó una valoración económica del trabajo doméstico y de cuidados no remunerados para el año 2017, detectando que éste "TDCNR" corresponde al $20 \%$ del PIB nacional para un total de más de 185.722 miles de millones de pesos, superando el valor agregado bruto de las actividades económicas más relevantes de la economía colombiana ${ }^{49}$.

Por otra parte, la dicotomía del trabajo productivo y reproductivo, por otra parte, no da cuenta de los roles que las mujeres desempeñan en el sector rural colombiano. Según el Informe sobre Desarrollo Humano del PNUD, se encontró que las mujeres representan la mayor parte de la mano de obra en el sector de la agricultura de subsistencia $y$, en promedio, un $43 \%$ del total de la población ocupada en la agricultura en los países en desarrollo ${ }^{50}$. Conforme a lo anterior, este paralelismo claramente se desconfigura, pues en efecto en el ámbito rural colombiano se ha evidenciado que las mujeres participan tanto en los trabajos de reproducción como de producción, es decir no sólo están a cargo de las tareas del cuidado doméstico que son infravaloradas, sino que además participan activamente en los procesos agrícolas de cultivo conel fin de sostener sus familias. De esta forma las mujeres, asignadas desde niñas en el rol de cuidadoras de la casa, se mueven también en las tareas de producción que sostienen la economía familiar ${ }^{51}$.

La conclusión de este apartado es que el colectivo de las mujeres rurales en Colombia se constituye como un grupo especialmente vulnerable/vulnerabilizado debido a que se enfrentan a las 5 caras de la opresión ${ }^{52}$ sostenidas en la teoría de Iris M. Young. Entendiendo la opresión, en sus términos, como estructural, la cual se refiere a los impedimentos sistemáticos que sufren algunos grupos y que sus causas están insertas en normas, hábitos y símbolos que no se cuestionan y que se reproducen en las instituciones económicas, políticas y culturales.

Las mujeres rurales viven la explotación como opresión en el sentido de que las reglas sociales les han asignado el rol del cuidado por el cual soportan una carga excesiva sumado también a los trabajos productivos y el sostenimiento de la economía familiar. Al no reconocer que el trabajo de cuidados, desempeñado mayoritariamente por las

\footnotetext{
49 "Información histórica - Cuenta Satélite de Economía del Cuidado (CSEC). Boletín Técnico" (DANE, 21 de agosto de 2020) <https://www.dane.gov.co/files/investigaciones/boletines/cuentas/ec/Bol CS Econ o cuidado matriz trabajo 2019.pdf> accedido el 8 de septiembre de 2021.

50 Informe de Desarrollo Humano 2019, Óp. Cit., p. 184.

${ }^{51}$ Ana Jimena Bautista Revelo y otras, Óp.Cit., p. 121.

52 Iris Marion Young, Faces of Opression (1979).
} 
mujeres, es una actividad que genera riqueza material de la sociedad, las mujeres viven la marginación como opresión, pues son expulsadas de la participación útil de la sociedad, quedando así potencialmente sujetas a graves privaciones materiales. Sumada a la marginación que se presenta por vivir en el campo y enfrentar mayores condiciones de precariedad en comparación con la población urbana. Las graves limitaciones que tienen las mujeres en el acceso a la tierra imposibilitan que se incremente su capacidad de actuación, su poder de toma de decisiones y su participación en el mercado de trabajo, por lo que se materializa la carencia de poder como opresión. Sufren el imperialismo cultural como opresión por la efectiva transposición de la supuesta división social del trabajo de las sociedades occidentales-patriarcales. Finalmente, las mujeres rurales padecen la más grave violencia como opresión, en todas sus modalidades, que se acentúa aún más en el marco del conflicto armado 53 .

\section{3.- Derechos: medidas diferenciales de género que contempla la Ley 1448 de 2011}

La Ley 1448 de 2011 se expide con el objetivo de "establecer un conjunto de medidas judiciales, administrativas, sociales y económicas, individuales y colectivas, en beneficio de las víctimas de las violaciones contempladas en el artículo 3 de la presente ley, dentro de un marco de justicia transicional, que posibiliten hacer efectivo el goce de sus derechos a la verdad, la justicia y la reparación con garantía de no repetición, de modo que se reconozca su condición de víctimas y se dignifique a través de la materialización de sus derechos constitucionales" (Art. 1).

Como ya expuso en el primer apartado, en concordancia con los desarrollos nacionales e internacionales referenciados, la ley incluye como uno de sus principios el enfoque diferencial: "El principio de enfoque diferencial reconoce que hay poblaciones con características particulares en razón de su edad, género, orientación sexual y situación de discapacidad". Por tal razón las medidas de protección, atención, asistencia y reparación integral que se establecen en la mencionada ley, contarán con dicho enfoque.

Así pues, en la Ley 1448 de 2011 se incorpora expresamente el enfoque diferencial- general y no sólo de género- a través del artículo 13. Este enfoque es reiterativo en los diferentes aspectos de la Ley 1448 , incluido lo referente a la restitución de tierras. Allí se establece, por ejemplo, como medida de protección, que para las mujeres víctimas del conflicto armado deberá tenerse en cuenta los riesgos

53 Ver: Women's link worldwide, Resumen Auto 092 de 2018. Riesgos de género en el marco del conflicto armado (2008) <https://www.womenslinkworldwide.org/files/1272/resumen-del-auto-0922008.pdf > accedido el 8 de septiembre de 2021. 
específicos, las modalidades de agresión y la vulnerabilidad para protegerse de sus agresores (Art 31, par. 3). En el marco de los procesos judiciales plantea medidas especiales como el deber de información, asesoría y apoyo, especialmente para las víctimas de delitos contra la integridad sexual (Art 35). También contempla una modalidad especial de testimonio (Art.41) y presencia de personal especializado (Art. 42). En las medidas de rehabilitación psicosocial, se resalta la importancia de la perspectiva de género y demanda la creación de un componente de atención psicosocial para las mujeres víctimas (Art. 136 y 137).

Con respecto a la restitución de tierras, la Ley 1448 en sus artículos 114 a 118 incluye unas normas especiales para las mujeres en los procesos de restitución de tierras y establece que el Estado debe dar especial protección a las mujeres víctimas de despojo o abandono forzado. Comprende principalmente la prelación en la atención en la etapa administrativa y judicial del proceso de restitución, también como la prioridad que se le debe otorgar a las mujeres en los beneficios que acompañan la restitución como crédito, adjudicación de tierras, garantías, seguridad social, educación, capacitación y recreación, subsidio familiar, planes y programas de reforestación y jornadas de cedulación.

Como medidas de protección para la mujer se encuentra la titulación conjunta, esto quiere decir, "que entodos los casos en que el demandante y su cónyuge, o compañero o compañera permanente, hubieran sido víctimas de abandono forzado y/o despojo del bien inmueble cuya restitución se reclama, el juez o magistrado en la sentencia ordenará que la restitución y/o la compensación se efectúen a favor de los dos y ordenará a la Oficina de Registro de Instrumentos Públicos que efectúe el respectivo registro a nombre de los dos, aun cuando el cónyuge o compañero o compañera permanente no hubiera comparecido al proceso" 54 .

Otra medida de protección que contempla la Ley 1448 de 2011 es la "obligación de consultar previamente a las mujeres sobre la posibilidad de contar con el acompañamiento de la Fuerza Pública en la diligencia de entrega material de los bienes, así como en el proceso de retorno, para garantizar las condiciones de seguridad y el goce efectivo de los derechos que tienen sobre el bien" 55 .

\footnotetext{
${ }^{54}$ Adriana Benjumea Rua y Natalia Poveda Rodríguez, El derecho a la tierra para las mujeres: una mirada a la ley de víctimas y restitución de tierras (Corporación Humanas. 2012) p. 72. 55 Ibid.
} 


\section{4.- Evaluando el impacto de género de la Ley 1448 de 2011 en las mujeres rurales}

En primer lugar, debe decirse que a pesar de que la Ley de Restitución de Tierras se desarrolla con el objetivo de lograr protección y reparación para las víctimas del conflicto armado dentro de un marco de justicia transicional, ésta en virtud de la figura de la reparación transformadora y el enfoque diferencial olvida la discriminación estructural a la que ha sido históricamente sometida la población campesina y en mayor medida las mujeres, como se ha demostrado a lo largo de la exposición. Esto quiere decir que más allá de lograr que las mujeres accedan con prioridad al proceso de restitución, la Ley de Restitución no alcanza a incorporar otras medidas de tipo estructural y permanente, más allá de las acciones afirmativas como único recurso, que de verdad materialicen y transversalicen el carácter reparador y transformador enunciados en los marcos normativos.

Asimismo, el artículo 13 establece el principio de enfoque diferencial, pero no el enfoque de género de manera concreta, con lo cual parece que éste haya quedado subsumido en dicha disposición normativa que fue formulada de manera amplia. Al respecto, en el Balance de la Ley 1448 realizado por la Procuraduría General de la Nación en el año 2020 se sostuvo que "esto es un abordaje complejo porque no aborda las relaciones de poder en los territorios. No hay una categoría que evidencia el enfoque de género como tal. Eso llevo a que en las instituciones se hablara de enfoque diferencial y se entendía como enfoque de género (ahí dentro). Desde ahí hay que hacer unas precisiones"56.

En materia de aplicación del enfoque de género en todo el proceso de restitución los principalesobstáculos para el acceso y efectiva restitución transformadora de las mujeres son:

- En la etapa administrativa, el reforzamiento de estereotipos de género en relación con el acceso de las mujeres a la tierra y un "enfoque familista", que se traducen por ejemplo en que las pruebas que recauda la URT tienden a demostrar los vínculos familiares de las mujeres con quienes tienen la titularidad del predio y no procuran establecer más bien cuáles son los vínculos jurídicos o económicos de las mujeres con la tierra ${ }^{57}$. Las mujeres no son consideradas titulares de la acción de restitución y no se reconoce su aporte productivo sobre

\footnotetext{
56 Procuraduría General de la Nación, Balance de la Ley 1448 de 2011. Componente de Enfoque Diferencial $y$ de género (2021) p. 25 $<$ https://www.procuraduria.gov.co/portal/media/file/PGN\%20Balance\%20Ley\%201 $448 \% 20$ de $\% 202011 \% 20$ Componente $\% 20$ enfoque $\% 20$ diferencial $\% 20 \mathrm{y} \% 20 \mathrm{de} \% 20 \mathrm{~g}$ $\%$ C3\%A9nero.pdf> accedido el 8 de septiembre de 2021.

57 Centro de Investigación y Educación Popular/ Programa por la Paz (CINEP/PPP), Género y Políticas de Tierras. Experiencias en cuatro países de países de América Latina (2016).
} 
los predios reclamados. No se incorporan "el enfoque diferencial de género en los instrumentos de recepción de la solicitud y en las metodologías para la documentación del proceso, por lo tanto, no se logra recoger y documentar de manera específica las facetas de género del desplazamiento y los daños específicos de las mujeres"58. Lo anterior implica que no se garantice el autoreconocimiento de las mujeres como pertenecientes a los grupos del enfoque diferencial, y con ello se les excluye de los beneficios de acceso prioritario a las medidas de la Ley. Además, no todos los funcionarios de la URT están capacitados para atender los casos de las mujeres, y si no tienen en cuenta sus condiciones de vulnerabilidad puede haber una revictimización ${ }^{59}$.

- En la etapa judicial, no se desarrollan ni se recogen elementos de género en el recaudo probatorio por parte de los operadores de justicia, y el incumplimiento de los términos procesales, debido a diversos factores como la complejidad del caso y el represamiento judicial, no corresponden con la necesidad de garantizar un acceso oportuno y preferente de las mujeres a la restitución ${ }^{60}$. En muchas ocasiones, no se toman en cuenta las afectaciones particulares de las mujeres cuando son víctimas de violencia sexual y esta es la causa principal del despojo y el desplazamiento, pues en el proceso judicial de restitución de tierras, la negación de esta vulnerabilidad implica que no se considere a la víctima como beneficiaria del programa de restitución argumentando que la pérdida del derecho a la tierra no está conectada con la situación de conflicto armado. La redacción de las demandas no se realiza con enfoque de género y por tanto no se solicita al juez que ordene medidas tendientes a cobijar las necesidades de las mujeres víctimas, en concordancia con lo dispuesto en la Ley 1448 de 2011, que incluyen medidas específicas sobre proyectos productivos, vivienda, líneas de crédito, educación, o la pretensión de acompañamiento de la fuerza pública para hacer entrega material del bien, entre otras 61

- En la etapa del fallo judicial, también se identifica un "enfoque familista" en "medidas como la inscripción en el registro del bien a nombre de ambos cónyuges o compañeros permanentes, a pesar de ser concebida como una disposición protectora que surge en un contexto donde los hombres, al aparecer como propietarios únicos,

\footnotetext{
58 Ibid., p. 148

59 Ibid., p. 145

60 International Land Coalition, Primer Informe Sombra específico de mujeres rurales y campesinas en Colombia presentado a la $72^{\circ}$ sesión del Comité de la Convención para todas las formas de discriminación contra la mujer CEDAW (2019) < https://www.semillas.org.co/es/otras-publicaciones/primer-informesombra-especi-769-fico-de-mujeres-rurales-y- campesinas-en-colombiapresentado-a-la-72o-sesio-769-n-del> accedido el 8 de septiembre de 2021.

61 Centro de Investigación y Educación Popular/ Programa por la Paz (CINEP/PPP), óp. Cit., p. 119.
} 
disponían de los bienes lo que de alguna manera defraudaba a la familia o a su pareja, lo real es que en los contextos de desplazamiento actuales, las mujeres en su mayoría son las que adelantan los procesos de reparación y restitución. Por lo anterior, la aplicación de la medida con el desconocimiento de estos contextos puede ocasionar que hombres que abandonaron de manera previa sus obligaciones económicas con hijos, hijas o parejas, aparezcan y se beneficien de un proceso en el cual las mujeres fueron las que asumieron los costos, riesgos y trámites"62. No se tiene en cuenta el artículo 116 de la Ley 1448 de 2011 sobre el pleno consentimiento previo de las mujeres al momento de la entrega de los predios y las medidas de seguridad complementarias.

- En la etapa posterior al fallo judicial, "las órdenes que restituyen los predios a favor del "núcleo familiar", o aquellas en las que se realiza la titulación conjunta a quienes actualmente no conviven juntos, limita el derecho a la libre disposición uso y goce de los bienes por parte de las mujeres, así como la libertad económica y de decisión sobre el predio"63. Se reportan también dificultades en la sostenibilidad de la tierra restituida cuando ésta se entrega con deudas de impuestos y servicios públicos y paralelamente no se implementan verdaderas reformas o políticas integrales que potencialicen la productividad rural y permitan el goce delderecho a la tierra de las mujeres de una manera digna y justa. En las entregas materiales de los bienes restituidos se generan conflictos con terceros que ocupan actualmente los predios, se han reportado casos en los que se permite la continuidad de proyectos agrícolas o extractivos en predios que están incluidos en procesos de restitución, de manera que cuando las personas finalmente regresan a sus tierras muchas veces ya existen concesiones 0 proyectos en ejecución, lo cual les impide disponer libremente de su propiedad ${ }^{64}$.

- Otra dificultad que se identifica es la falta de datos diferenciados con respecto al acceso al proceso de restitución de tierras, "a la fecha no existe un programa de titulación de predios que determine el número de mujeres beneficiarias, la cantidad de hectáreas a entregar, las condiciones de los predios y el periodo en el cual se realizará esta entrega. El Estado no cuentacon un análisis de la materialización de las sentencias de restitución, hace balances únicamente de los fallos o de los procesos. No indaga respecto al número de personas que hansido restituidas formalmente y que se encuentran actualmente habitando, explotando o disponiendo sus predios, y mucho menos un examen respecto de las mujeres rurales que se encuentran recibiendo de manera articulada los beneficios

\footnotetext{
62 Adriana Benjumea Rúa y Natalia Poveda Rodríguez, Óp. Cit., p. 72

${ }^{63}$ Centro de Investigación y Educación Popular/ Programa por la Paz (CINEP/PPP), Óp. Cit., p. 152.

${ }^{64}$ International Land Coalition, Op. Cit., p. 16
} 
propios de la restitución"65. Bajo esta misma línea los mecanismos de implementación de la Ley no cuentan con sistemas de información, monitoreo y evaluación que incluyen variables para el análisis de género sobre el impacto de la Ley y sus programas en todas las etapas de los procesos. Es imprescindible también resaltar la falta de participación de las mujeres rurales, en la formulación e implementación de los proyectos y programas de las que son beneficiarias. No son consultadas, ni juegan un rol protagónico y participativo, sino que se les concibe sólo como sujetos pasivos y beneficiarias de la oferta institucional. Tampoco se identifican las barreras estructurales que evitan que haya una participación efectiva de las mujeres, ni se coadyuva en su superación. Barreras tales como la falta de conocimientos y capacidades para representar los intereses respectivos ${ }^{66}$, o la excesiva carga laboral de cuidado ${ }^{67}$, que les impide usar su tiempo en las mesas de participación de víctimas.

\section{5.- Rediseñando la Ley 1448 de 2011}

Con el objeto de resaltar buenas prácticas en materia de restitución de tierras a las mujeres rurales y con el fin de aprovechar la experiencia que el desarrollo normativo y la implementación de Ley 1448 de 2011 ha otorgado de cara a la nueva Reforma Rural Integral que se establece mediante el punto número 1 del Acuerdo de Paz, se proponen las siguientes recomendaciones:

- En la etapa administrativa del proceso de restitución de tierras, el registro único de víctimas creado por la Ley 1448 debe ser sensible al género y en este sentido concebir las específicas violaciones a los derechos humanos que afectan a las mujeres. Por esta razón, al momento de diligenciar el registro debe transcribirse la declaración integral y completa realizada por la mujer e de forma independiente de las declaraciones de otros interesados en los mismos predios, inclusive del mismo núcleo familiar. También debe tenerse en cuenta que la violencia sexual no es la única modalidad de violencia de género de las que han sido víctimas las mujeres. Por lo cual no deben invisibilizarse otras violencias basadas en el género y debe haber un acercamiento con enfoque diferencial a los hechos victimizantes narrados por las mujeres. Además, es esencial la aplicación de pruebas comunitarias

\footnotetext{
${ }^{65}$ Ibid., p. 31.

${ }^{66}$ Este tipo de brecha se zanja con programas de capacitación de conocimientos técnicos, conceptuales, legales y políticos de los enfoques diferenciales para fortalecer los liderazgos sociales de las víctimas.

67 Ver por ejemplo que en el Protocolo de Participación en materia de garantías de participación para los sujetos de especial protección constitucional y/o sus representantes, está contemplado el apoyo a las mujeres víctimas con hijos menores de 5 años que consiste principalmente en el servicio de hogares infantiles del ICBF.
} 
para esclarecer los hechos de violencia que permitieron o facilitaron el despojo o abandono forzado. Por otra parte, establecer la conformación de grupos familiares y de los roles o actividades que las mujeres desempeñan al interior de su comunidad, como la contribución de éstas en las actividades relacionadas con la tierra. Garantizar el acompañamiento psicosocial durante todas las etapas del proceso y promover el desarrollo de procesos de formación y sensibilización de funcionarios involucrados en la restitución de tierras ${ }^{68}$. Promover espacios de confidencialidad en la infraestructura física de la URT para la recepción de las declaraciones.

- En la etapa judicial, es importante reconocer que en el sector rural la propiedad de la mayoría de los predios no se encuentra formalizada, por tanto, los jueces deben reconocer los diferentes procesos de ocupación, uso y tenencia de la tierra por parte de las mujeres y en esa medida flexibilizar las pruebas llamadas a demostrar los vínculos jurídicos y económicos de las mujeres con los predios solicitados, y dejar constancia de esta vinculación en la parte considerativa de las sentencias. Reconocer también en las sentencias de restitución las afectaciones particulares de las mujeres en el contexto del conflicto armado, como ha explicado reiteradamente la Corte Constitucional a través de distintos autos y sentencias. Ordenar incluir a las mujeres como titulares en las herramientas de información jurídica (catastro, folios de matrícula, resoluciones de adjudicación, entre otras), y también su inclusión en los programas complementarios a la restitución (salud, educación, vivienda, préstamos, proyectos productivos, entre otros), aunque haya sido el compañero permanente o cónyuge el que haya iniciado el proceso.

- En la etapa posterior al fallo judicial, el Estado debe hacerse cargo de pagar las deudas por concepto de impuestos y servicios públicos generadas durante el desplazamiento forzado. Determinar algunas garantías frente a la independencia económica de la mujer, por ejemplo, a través del apoyo financiero y tecnológico de proyectos productivos u otras formas de generar ingresos ${ }^{69}$. Garantizar también la libertad de uso, goce y disposición del predio que se haya titulado o formalizado a su nombre, y esto implica que se resuelvan los asuntos que puedan perturbar la posesión de las beneficiarias sobre el predio, adelantar diligencias de desalojo integrales a terceros que estén ocupando los terrenos y el acompañamiento de la Fuerza Pública en la

68 Centro de Investigación y Educación Popular/ Programa por la Paz (CINEP/PPP), Óp. Cit., p. 145

${ }^{69}$ Comité para la Eliminación de la Discriminación contra la Mujer, Observaciones finales sobre el noveno informe periódico de Colombia (2019) <https://www.refworld.org.es/pdfid/5ce587b24.pdf> accedido el 8 de septiembre de 2021. 
entrega material del bien, siempre y cuando las mujeres hayan dado previo consentimiento para ello ${ }^{70}$.

- Otras medidas necesarias que garanticen el enfoque de género de forma integral en el marco de la ley de restitución de tierras son: adoptar e implementar indicadores de género en los sistemas estadísticos que generen información cualitativa acerca de la propiedad de la tierra y el control de los recursos productivos, como la tierra, el agua, los equipos, los insumos, la información y el crédito, así como del acceso a estos, con el fin de obtener líneas base que especifiquen formas de violencias y de discriminaciones y de trabajo no remunerado vinculado a la economía del cuidado de mujeres y niñas rurales y campesinas y para que esta información se tenga en cuenta en el diseño con enfoque de género de los programas de adjudicación de tierras y entrega de títulos de propiedad, entre otros. Garantizar la participación de las mujeres rurales y de sus organizaciones en las fases de diseño, implementación, ejecución, seguimiento y evaluación de la Ley de víctimas y en la construcción de los planes de desarrollo territorial para que se escuchen e incorporen sus reclamaciones y propuestas. Para ello es necesario tener en cuenta las necesidades específicas y condiciones diferenciales de las mujeres, de acuerdo con su ciclo vital, afectaciones y necesidades, así como la identificación de las principales barreras que impiden su participación y la superación de las mismas a través de diferentes iniciativas gubernamentales. Así mismo "los proyectos de restitución de tierras y patrimonio deben valorar el aporte de las mujeres en la reconstrucción de la memoria del territorio afectado por el conflicto armado, y en las cartografías comunitarias que se realicen"71.

\section{3.- BIBLIOGRAFÍA}

Adriana Benjumea Rúa y Natalia Poveda Rodríguez, El derecho a la tierra para las mujeres: una mirada a la ley de víctimas $y$ restitución de tierras (Corporación Humanas 2012).

Ana Jimena Bautista Revelo y otros, Voces Desde El Cocal. Mujeres Que Construyen Territorio (De Justicia 2018).

Asamblea General de las Naciones Unidas, Pacto Internacional de Derechos Económicos, Sociales y Culturales (1966) $<$ www.ohchr.org/sp/professionalinterest/pages/cescr.aspx > accedido el 8 de septiembre de 2021.

Asamblea General de las Naciones Unidas, Convención sobre la eliminación de todas las formas de discriminación contra la mujer (1979)

${ }^{70}$ Centro de Investigación y Educación Popular/ Programa por la Paz (CINEP/PPP), Óp. Cit., p. 119

${ }^{71}$ Comisión Colombiana de Juristas, Óp. Cit., p. 45. 
<www.ohchr.org/sp/professionalinterest/pages/cedaw.aspx > accedido el 8 de septiembre de 2021.

Asamblea General de las Naciones Unidas, Declaración de las Naciones Unidas sobre los Derechos de los Campesinos y de Otras Personas que Trabajan en las Zonas Rurales (2018) $<$ www.ohchr.org/Documents/HRBodies/HRCouncil/WGPleasants $\angle A-H R C-W G-15-1-2$ sp.pdf $>$ accedido el 8 de septiembre de 2021.

Asamblea General de las Naciones Unidas, Pacto Internacional de Derechos Civiles y Políticos (1976) <www.ohchr.org/Documents/ProfessionalInterest/ccpr SP.pdf> accedido el 8 de septiembre de 2021.

Auto 092/2008, Corte Constitucional, 14 de abril de 2008 (Colombia) $<$ www.corteconstitucional.gov.co/relatoria/autos/2008/a09208.htm > accedido el 8 de septiembre de 2021.

Auto 218/2006, Corte Constitucional, 11 de agosto de 2006 (Colombia) $<$ www.corteconstitucional.gov.co/T-02504/AUTOS\%202006/26.\%20Auto\%20del\%2011-082009.\%20Auto\%20218.\%20Verificacion\%20medidas.PDF> accedido el 8 de septiembre de 2021.

CCdJ, La política agraria y los derechos de las mujeres en Colombia (2011)

$<$ www.coljuristas.org/documentos/libros e informes/la politica agraria y los derechos de las mujeres.pdf $>$ accedido el 8 de septiembre de 2021.

Censo Nacional de Población y Vivienda (DANE, 10 de octubre de 2018) $<$ www.dane.gov.co/> accedido el 8 de septiembre de 2021.

Centro de Investigación y Educación Popular/ Programa por la Paz (CINEP/PPP), Género y Políticas de Tierras. Experiencias en cuatro países de países de América Latina (2016).

Consejo Económico y Social, Resolución de la Comisión de Derechos Humanos 2000/13. La igualdad de las mujeres en materia de propiedad, acceso y control de la tierra y la igualdad de derechos a la propiedad y a una vivienda adecuada (2000) $<$ www.acnur.org/fileadmin/Documentos/BDL/2002/0653.pdf?fil $\mathrm{e}=$ fileadmin/Documentos/BDL/2002/0653.Numeral\%205. $>$ accedido el 8 de septiembre de 2021.

Constitución Política de Colombia, 13 de junio de 1991 $<$ www.secretariasenado.gov.co/senado/basedoc/constitucion p olitica_1991.html> accedido el 8 de septiembre de 2021.

En Caquetá Tiene Más Derechos Una Vaca Que Una Mujer: La Lucha Por La Tierra De Las Lideresas Campesinas En Colombia' (2021) Mongabay- Periodismo Ambiental Independiente en América Latina <https://es.mongabay.com/2021/03/en-caqueta-tienemas-derechos-una-vaca-que-una-mujer-la-lucha-por-la-tierrade-las-lideresas-campesinas-en-colombia/> accedido el 8 de septiembre de 2021. 
Encuesta de cultura política (ECP) DANE (DANE, 8 de noviembre de 2019) $<$ www.dane.gov.co/index.php/comunicados-yboletines/estadisticas-sociales/cultura-politica> accedido el 8 de septiembre de 2021.

Encuesta nacional del Uso del Tiempo (DANE, 21 de junio de 2021) $<$ https://www.dane.gov.co/index.php/estadisticas-por-tema2/pobreza-y-condiciones-de-vida/encuesta-nacional-del-usodel-tiempo-enut> accedido el 8 de septiembre de 2021.

Infografía Las Mujeres Rurales en América Latina (International Land Coalition, 12 de diciembre de 2018) <https://lac.landcoalition.org/es/recursos/infograf\%C3\%ADalas-mujeres-rurales-en-am\%C3\%A9rica-latina/> accedido el 8 de septiembre de 2021.

Información histórica - Cuenta Satélite de Economía del Cuidado (CSEC). Boletín Técnico" (DANE, 21 de agosto de 2020)<https://www.dane.gov.co/files/investigaciones/boletines /cuentas/ec/Bol_CS Econo cuidado matriz trabajo 2019.pdf> accedido el 8 de septiembre de 2021.

International Land Coalition, Primer Informe Sombra específico de mujeres rurales y campesinas en Colombia presentado a la $72^{\circ}$ sesión del Comité de la Convención para todas las formas de discriminación contrala mujer CEDAW (2019) $<$ https://www.semillas.org.co/es/otras-publicaciones/primerinforme-sombra-especi-769-fico-de-mujeres-rurales-ycampesinas-en-colombia-presentado-a-la-720-sesio-769-n-del> accedido el 8 de septiembre de 2021.

Iris Marion Young, Faces of Opression (1979)

Ley 1448 de 2011, Ley, 10 de junio de 2011 (Colombia) <http://wp.presidencia.gov.co/sitios/normativa/leyes/Document s/Juridica/LEY\%201448\%20DE\%202011.pdf> accedido el 8 de septiembre de 2021.

Ley 2078 de 2021, Ley, 8 de enero de 2021 (Colombia) <https://dapre.presidencia.gov.co/normativa/normativa/LEY\%2 02078\%20DEL\%208\%20DE\%20ENERO\%20DE\%202021.pdf> accedido el 8 de septiembre de 2021.

Nota estadística: Mujeres Rurales en Colombia (DANE, 27 de noviembre de 2020) <www.dane.gov.co/index.php/estadisticaspor-tema/enfoque-diferencial-e-interseccional/enfoque-degenero > accedido el 8 de septiembre de 2021.

Organización de las Naciones Unidas, Principios sobre la restitución de las viviendas y el patrimonio de los refugiados y las personas desplazadas (2007).

$<$ www.acnur.org/fileadmin/Documentos/Publicaciones/2008/63 25.pdf $>$ accedido el 8 de septiembre de 2021.

OXFAM, Radiografía de la desigualdad. Lo que nos dice el último censo agropecuario sobre la distribución de la tierra en Colombia (2017) <https://oi-files-d8-prod.s3.eu-west-2.amazonaws.com/s3fs- 
public/file attachments/radiografia de la desigualdad.pdf> accedido el 8 de septiembre de 2021.

Procuraduría General de la Nación, Balance de la Ley 1448 de 2011. Componente de Enfoque Diferencial y de género (2021) p. 25 $<$ https://www.procuraduria.gov.co/portal/media/file/PGN\%20B alance $\% 20$ Ley $\% 201448 \% 20$ de $\% 202011 \% 20$ Componente $\% 20 \mathrm{e}$ nfoque $\% 20$ diferencial\%20y\%20de\%20g\%C3\%A9nero.pdf> accedido el 8 de septiembre de 2021.

Procuraduría General de la Nación, Tercer Informe al Congreso sobre el estado de Avance de la Implementación del Acuerdo de Paz. Abril 2020-Marzo 2021. Conclusiones y recomendaciones. (2021)<https://www.procuraduria.gov.co/portal/media/file/Proc uraduri\%CC $\% 81 \mathrm{a} \% 20$ Conclusiones $\% 20 \mathrm{y} \% 20 \mathrm{recomendaciones}$ \%20Tercer\%20Informe\%20Paz\%202021.pdf> accedido el 8 de septiembre de 2021.

Programa de las Naciones Unidas para el Desarrollo, Informe de Desarrollo Humano 2019

(2019) <http://hdr.undp.org/sites/default/files/hdr_2019_overview__spanish.pdf> accedido el 8 de septiembre de 2021>

Programa de las Naciones Unidas para el Desarrollo, Informe Nacional de Desarrollo Humano(2011)<www1.undp.org/content/undp/es/home/library page/hdr/human developmentreport2011.html> accedido el 8 de septiembre de 2021.

Recomendación general núm. 34 (2016) sobre los derechos de las mujeres rurales (CEDAW/C/GC/34, $<$ www.acnur.org/fileadmin/Documentos/BDL/2016/10709.pdf?fi le=fileadmin/Documentos/BDL/2016/10709> accedido el 8 de septiembre de 2021.

Registro Único de Víctimas (Unidad para la Reparación y Atención Integral a las Víctimas, 31 de julio de 2021) $<$ www.unidadvictimas.gov.co/es/registro-unico-de-victimasruv/37394> accedido el 8 de septiembre de 2021.

Sentencia T024-2004, Corte Constitucional, 21 de enero de 2004 (Colombia) <www.corteconstitucional.gov.co/relatoria/2004/T024-04.htm> accedido el 8 de septiembre de 2021.

Women's link worldwide, Resumen Auto 092 de 2018. Riesgos de género en el marco del conflicto armado (2008) $<$ https://www.womenslinkworldwide.org/files/1272/resumendel-auto-092-2008.pdf> accedido el 8 de septiembre de 2021. 